\title{
Oxidative Stress, Altered-Self and Autoimmunity
}

\author{
Ivan C. Gerling* \\ University of Tennessee Health Science Center, and Veterans Affairs Medical Center, Research Service 151, 1030 \\ Jefferson Avenue, Memphis, TN 38104, USA
}

\begin{abstract}
It is well known that oxidative stress can induce production of free radicals that can modify proteins. The hypothesis presented here is that the target antigens in autoimmune diseases, such as type 1 diabetes mellitus, are not normal self proteins but rather self proteins that have been altered by free radicals, produced as a result of oxidative stress in the target cells. The observation that disease often occurs only in a single tissue, even when other tissues contain the same antigen, could be explained if both the presence of the antigen and severe oxidative stress is required, before tissue destruction occurs. Furthermore, the cyclic nature of tissue destruction and problems with detecting robust high affinity autoreactivity to self antigens may be easier to understand if we presume that pathological autoreactivity is targeting redoxmodified self antigens, while assays to unmodified self only measure its cross-reactivity to normal self antigens.
\end{abstract}

\section{BACKGROUND}

Autoimmune diseases such as type 1 diabetes mellitus (DM1) are believed to result from the failure of immunological tolerance to protein self antigens. It has been proposed that alterations in self antigens could initiate the process of autoimmunity $[1,2]$. According to this hypothesis one does not need to assume that autoimmune diseases are initiated exclusively by imperfections in the ability of the immune system to tolerize itself to normal cellular components (self antigens). Instead, the self antigens change to a previously unseen form and, therefore, become a natural target of the immune system. The immune response to the altered self antigen then progresses and helps develop immunity to unaltered self antigens as well.

If the mitochondria are dysfunctional or cells are under stress, such as during high metabolic demand, viral infections, or exposure to certain cytokines/toxins, cells may produce a sufficient amount of radical oxygen or radical nitrogen species (ROS/RNS) to overwhelm the antioxidant systems that normally neutralize these free radicals [3]. These ROS/RNS are very short-lived because they react with and modify, lipids and proteins in the cell [4]. The field of redox proteomics has been progressing rapidly in the past decade and has developed new methods that allow investigators to study a large number of specific modifications of proteins induced by free radicals [5-7]. The reaction of ROS/RNS with proteins can create stable end products such as 3chlorothyrosine and 3-nitrotyrosine that may not only block natural modifications of the tyrosine by e.g., phosphorylation, but also change the antigenic profile of the protein. The reaction of hydrogen peroxide with the chloride anion can create hypochlorous acid, which deaminates serine and con-

\footnotetext{
*Address correspondence to this author at the University of Tennessee Health Science Center, and Veterans Affairs Medical Center, Research Service 151, 1030 Jefferson Avenue, Memphis, TN 38104, USA; Tel: 1901-523-8990, Ext: 5088; Fax: 1-901-577-7273;

E-mail: igerling@utmem.edu
}

verts it into glycoaldehyde. These aldehydes not only change the antigenic profile of a protein, but actually enhance the antigenicity of proteins, turning non-immunogenic malaria antigens into effective immunogens [8].

A number of oxidative protein modification have been described in autoimmune diseases [9]. In systemic lupus erythematosus the $60 \mathrm{kD}$ Ro ribonucleoprotein is one of several autoantigenic targets. Autoimmunity was initiated at an accelerated pace in animals immunized with oxidatively modified 60kD Ro protein [10]. Oxidative modifications produced high molecular weight complexes of glutamic acid decarboxylase (GAD) and sera from type 1 diabetic patients bound these complexes much more strongly than the monomer GAD autoantigen [11]. It has been shown that several of the autoantigens targeted in diffuse scleroderma are uniquely susceptible to cleavage by ROS [12]. Oxidation of beta-2glycoprotein, a target of antiphospholipid antibodies with hydrogen peroxide rendered this protein able to activate immature monocyte-derived dendritic cells [13].

It has been shown that the insulin-producing beta cells in the islet of Langerhans are particularly vulnerable to damage by free radicals [14]. Furthermore, beta cells appear to express relatively low levels of some antioxidants and are slow to increase their expression in response to oxidative stress [15]. That would leave the beta cells particularly susceptible to modifications on their proteins when ROS/RNS production is increased. Metabolic, infectious, or other types of stress on the cells could, therefore, result in substantial levels of redox modification on proteins in the cells (Fig. 1).

\section{HYPOTHESIS}

The hypothesis presented here is that: the targets of pathologic immune responses in autoimmune diseases such as DM1 are not normal self proteins but rather self proteins that have been altered by ROS/RNS, produced as a result of oxidative stress in the target cells. The principal challenge to 


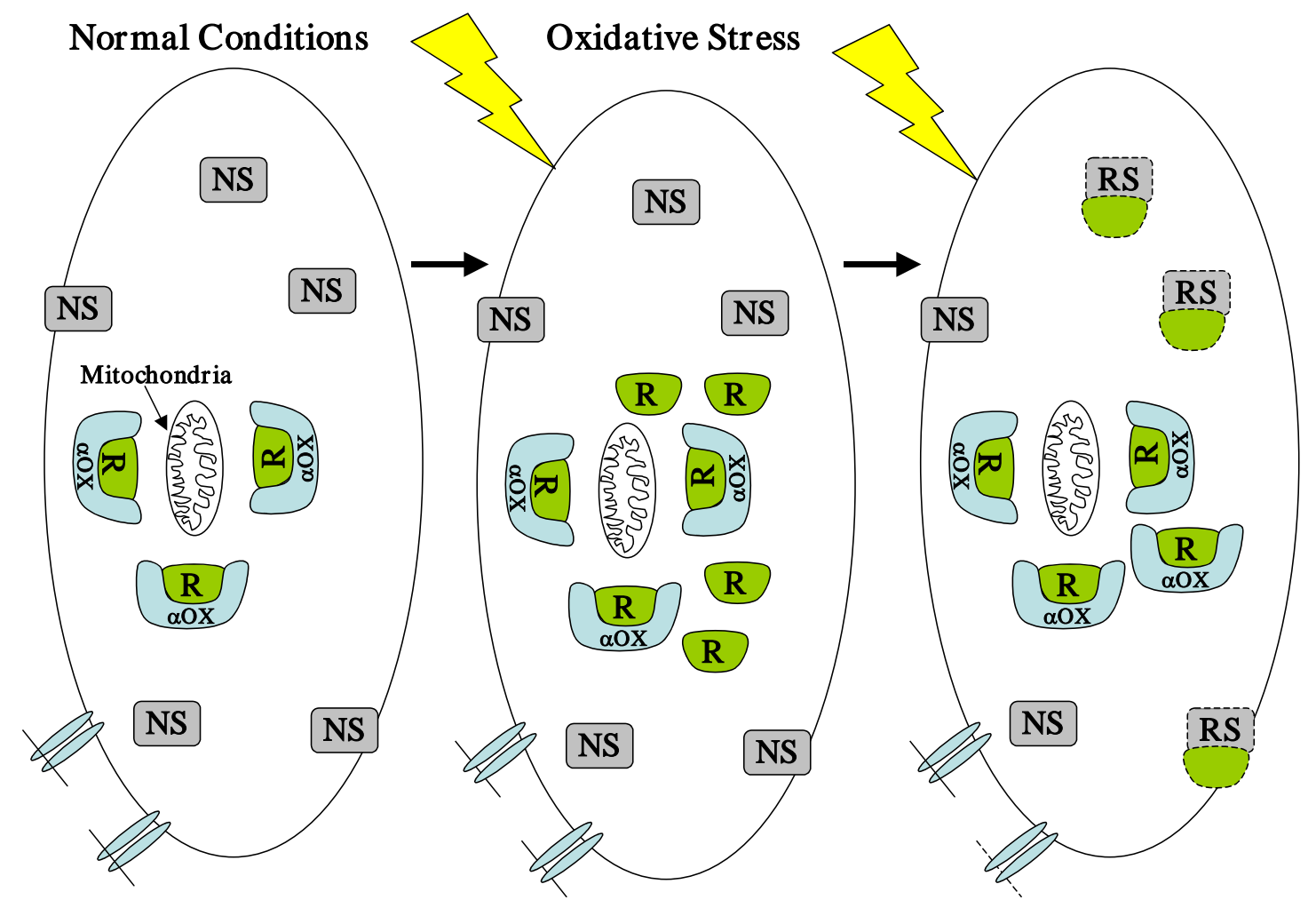

Fig. (1). Alteration of self proteins by oxidative stress. Mitochondria inside a cell produces free radicals (R) that are neutralized by antioxidant defenses $(\alpha \mathrm{OX})$ such that normal self antigens (NS) remain protected from damage by free radicals. Under conditions of increased free radical production (oxidative stress) the antioxidant defenses may not be able to neutralize all the free radicals produced. As a result, some of the normal self antigens are targeted by free radicals and become radical modified self antigens (RS) structures that the immune system may not have been tolerized to and, therefore, will develop responses against. The altered self recognized by the immune system could either be expressed directly on the surface of the cell or as peptide fragments of internal antigens presented by MHC class I molecules on the surface of the cell.

this hypothesis is to explain the fact that assays used to detect autoimmune responses often use antigens isolated from normal tissues or normal proteins/peptides. However, a considerable amount of stress is induced on tissues such as islets of Langerhans during their isolation. Furthermore, synthetic peptides and purified/recombinant proteins often have prolonged exposure to the oxygen in normal air and could undergo oxidizing modifications as a result of that exposure. Therefore, a small fraction of the purified normal self antigens could be redox-modified. That small fraction could provide a signal for "autoreactivity" in assays of reactivity to (presumed) normal-self. Even if the autoantigen preparations used are completely devoid of redox modifications, an immune response to modified self could have enough cross reactivity to the corresponding normal self epitopes that it would give a signal in some of the autoreactivity assays, even if that reactivity is not strong enough to be of pathophysiological relevance. This would be particularly likely to occur with detection of autoantibodies, because the requirement is just for sufficient binding affinity to not be "washed off" by a particular buffer.

While this hypothesis initially seems fairly easy to test, it requires careful consideration of experimental designs and limitations. It should not be presumed that we are dealing with an all-or-none phenomenon, but rather that there is some cross reactivity between the normal self antigen and the redox-modified self antigen. The immune response to normal self is presumed to be sufficiently lower than that to redox-modified self to render the former of little or no pathophysiological consequence. Yet any preparation of normal self antigen and redox-modified self antigen is unlikely to be $100 \%$ pure and will most likely contain a mixture of both. Fortunately there are now fairly reliable methods to quantitate the amount of most redox modifications [16] and self antigens, thus for each specific preparation it should be possible to define its relative content of both the modified and unmodified forms of the autoantigen. Combined with traditional titration of binding affinities it should be possible to show if there is indeed a stronger "autoreactivity" to self antigens and self epitopes if they have undergone redox modifications. Another complication is the large number of different modifications that can be induced by ROS/RNS. Because each modification will change the molecular bulk of the antigen in different ways, each may have to be studied separately.

\section{DISCUSSION}

One implication of this hypothesis is that immunological tolerance to true self antigens is never broken in autoimmune diseases such as DM1, and that idea may appear somewhat provocative. However, this assumption may help explain certain clinical phenomena in DM1 that have been somewhat difficult to understand otherwise. It is well known that after initiation of treatment with insulin in newly diagnosed DM1 
patients their production of C-peptide and needs for insulin may decrease, even to the point of making some patients insulin-independent for up to a year after initial diagnosis (the honeymoon phenomenon). This increase in the patient's own insulin production suggests that the immune destruction of beta cells diminishes after initiation of insulin therapy. Such temporary reductions in immune responses, even though the antigen is still present, have not been reported for other types of immune responses. Indeed, it has generally been found that if a virus or bacteria is re-introduced, the secondary immune response occurs both faster and with greater magnitude than the original response. The honeymoon phenomenon may be easier to understand in the context of the hypothesis presented here. In the period before diagnosis of DM1, beta cells are under severe metabolic stress because a dwindling number of these cells tries to keep up with a constant demand for insulin. However, after the initiation of insulin injections in the patient, this metabolic demand, on remaining or newly generated beta cells, would be drastically reduced, as would their ROS/RNS production. As a result, the remaining beta cells would have a drastic reduction in the amount of redox-modified self antigens they express, and become much less of a target for an immune response to redox-modified self antigens. Yet, the inevitable imperfections in insulin therapy would eventually expose the remaining islets to high glucose and the accompanying oxidative stress. Therefore, the honeymoon phenomenon may be easier to explain in the context of immunity to redoxmodified altered self than in the context of an immune response to true self antigens. It should also be noted that a substantial number of patients diagnosed with type 2 diabetes have been shown to have autoantibodies in their plasma $[17,18]$. Although the original pathology in type 2 diabetes is not related to autoimmune destruction of beta cells, it is certainly possible that oxidative stress and metabolic demand on beta cells in these patients could provoke development of pathogenic beta cell immunity to redox modified autoantigens, as a later complication.

Although many lines of evidence suggest a central role for $\mathrm{T}$ lymphocytes in DM1, it has been difficult to define beta cell antigens and epitopes with strong signals of T-cell autoreactivity in blood from DM1 patients. Stimulation indexes and other measures of T-cell autoreactivity are often considerably smaller that what is seen in measurements of other forms of T-cell reactivity [19-21]. That problem may be easier to understand in the context of this hypothesis. The T-cell receptor is presented with a small peptide fragment of its autoantigen [22], and the presence or absence of an oxidative modification could represent a substantial difference in the molecular "bulk" of such a small molecule. As a result, the binding of a T-cell receptor that is specific to a redoxmodified peptide would likely be considerably weaker if it was presented with the non-modified form of the same peptide.

The hypothesis that altered self is the target in organspecific autoimmune diseases, such as DM1, could also help explain why the autoimmune responses are organ-specific and do not attack all tissues that express the target antigens. A tissue would only be targeted if it both expresses the autoantigens and also is undergoing a level of oxidative stress that overwhelms its antioxidant defences. Furthermore, depending on the reason for oxidative stress it may not be constantly present in a given tissue, leading to periods of more or less activity of the disease.

Many years ago it was reported that when serum from newly diagnosed DM1 children were used to immunoprecipitate the $64 \mathrm{kD}$ autoantigen (later identified as GAD) from mouse islet of Langerhans, the immunoprecipitated band was more intense if the islets came from mice infected with an islet tropic strain of Coxsackie virus B4 [23]. At that time it was concluded that expression of the $64 \mathrm{kD}$ autoantigen in the islets had been increased by the virus infection. However, in light of the above hypothesis an equally possible explanation would be that the infection could have increased oxidative stress and redox-modified GAD in the islets that could be immunoprecipitated by the DM1 patient serum.

From a treatment point of view this hypothesis would suggest that, in addition to therapies targeting the immune system, we should also consider developing therapies that decrease oxidative stress in the target tissue. Such therapy could be used both for prevention and early treatment of the disease. Furthermore, tolerance inducing therapy should be using redox-modified autoantigens rather than normal autoantigens to be effective.

\section{ACKNOWLEDGEMENTS}

The author acknowledges grant support from National Institute of Health (DK062103) and editing assistance from Mr. Richard A. Parkinson.

\section{ABBREVIATIONS}

DM1
ROS
RNS
GAD 1 Radical Oxygen Species
Radical Nitrogen Species

\section{REFERENCES}

[1] Gordon EE. Altered oligosaccharides as the initiating autoantigen in rheumatoid arthritis. Med Hypotheses 1983; 10: 347-52.

[2] Abu-Shakra M, Shoenfeld Y. Parasitic infection and autoimmunity. Autoimmunity 1991; 9: 337-44.

[3] Djordjevic VB. Free radicals in cell biology. Int Rev Cytol 2004; 237: 57-89.

[4] Dalle-Donne I, Scaloni A, Giustarini D, et al. Proteins as biomarkers of oxidative/nitrosative stress in diseases: the contribution of redox proteomics. Mass Spectrom Rev 2005; 24: 55-99.

[5] Fratelli M, Demol H, Puype M, et al. Identification by redox proteomics of glutathionylated proteins in oxidatively stressed human T lymphocytes. Proc Natl Acad Sci USA 2002; 99: 3505-10.

[6] Ghezzi P, Bonetto V. Redox proteomics: identification of oxidatively modified proteins. Proteomics 2003; 7 : 1145-53.

[7] Zhan X, Desiderio DM. The human pituitary nitroproteome: detection of nitrotyrosyl-proteins with two-dimensional Western blotting, and amino acid sequence determination with mass spectrometry. Biochem Biophys Res Commun 2004; 325: 1180-6.

[8] Allison ME, Fearon DT. Enhanced immunogenicity of aldehydebearing antigens: a possible link between innate and adaptive immunity. Eur J Immunol 2000; 10: 2881-7.

[9] Kurien BT, Scofield RH. Autoimmunity and oxidatively modified autoantigens. Autoimmun Rev 2008; 7: 567-73.

[10] Scofield RH, Kurien BT, Ganick S, et al. Modification of lupusassociated $60-\mathrm{kDa}$ Ro protein with the lipid oxidation product 4hydroxy-2-nonenal increases antigenicity and facilitates epitope spreading. Free Radic Biol Med 2005; 38: 719-28.

[11] Trigwell SM, Radford PM, Page SR, et al. Islet glutamic acid decarboxylase modified by reactive oxygen species is recognized by 
antibodies from patients with type 1 diabetes mellitus. Clin Exp Immunol 2001; 126: 242-9.

[12] Casciola-Rosen L, Wigley F, Rosen A. Scleroderma autoantigens are uniquely fragmented by metal-catalyzed oxidation reactions: implications for pathogenesis. J Exp Med 1997; 185: 71-9.

[13] Buttari B, Profumo E, Mattei V, et al. Oxidized beta2-glycoprotein I induces human dendritic cell maturation and promotes a $\mathrm{T}$ helper type 1 response. Blood 2005; 106: 3880-7.

[14] Gandy SE, Buse MG, Crouch RK. Protective role of superoxide dismutase against diabetogenic drugs. J Clin Invest 1982; 70: 6508.

[15] Tiedge M, Lortz S, Drinkgern J, Lenzen S. Relation between antioxidant enzyme gene expression and antioxidative defense status of insulin-producing cells. Diabetes 1997; 46: 1733-42.

[16] Dalle-Donne I, Scaloni A, Butterfield DA, Eds. Redox Proteomics: From Protein Modifications to Cellular Dysfunction and Diseases. USA: Wiley-Interscience 2006.

[17] Fourlanos S, Dotta F, Greenbaum CJ, et al. Latent autoimmune diabetes in adults (LADA) should be less latent. Diabetologia 2005; 48: 2206-12.
[18] Davies H, Brophy S, Fielding A, et al. Latent autoimmune diabetes in adults (LADA) in South Wales: incidence and characterization. Diabet Med 2008; 25: 1354-7.

[19] Atkinson MA, Bowman MA, Campbell L, Darrow BL, Kaufman DL, Maclaren NK. Cellular immunity to a determinant common to glutamate decarboxylase and coxsackie virus in insulin-dependent diabetes. J Clin Invest 1994; 94: 2125-9.

[20] Roep BO. Autoreactive T cells in endocrine/organ-specific autoimmunity: why has progress been so slow? Springer Semin Immunopathol 2002; 24: 261-71.

[21] Zhang L, Nakayama M, Eisenbarth GS. Insulin as an autoantigen in NOD/human diabetes. Curr Opin Immunol 2008; 20: 111-8.

[22] Larbi A, Fülöp T, Pawelec G. Immune receptor signaling, aging and autoimmunity. Adv Exp Med Biol 2008; 640: 312-24.

[23] Gerling I, Nejman C, Chatterjee NK. Effect of coxsackievirus B4 infection in mice on expression of 64,000-Mr autoantigen and glucose sensitivity of islets before development of hyperglycemia. Diabetes 1988; 37: 1419-25.

(C) Ivan C. Gerling; Licensee Bentham Open.

This is an open access article licensed under the terms of the Creative Commons Attribution Non-Commercial License (http: //creativecommons. org/licenses/ by-nc/3. 0/) which permits unrestricted, non-commercial use, distribution and reproduction in any medium, provided the work is properly cited. 